\title{
Thomas Handbuch
}

\author{
Hrsg. v. Volker Leppin
}

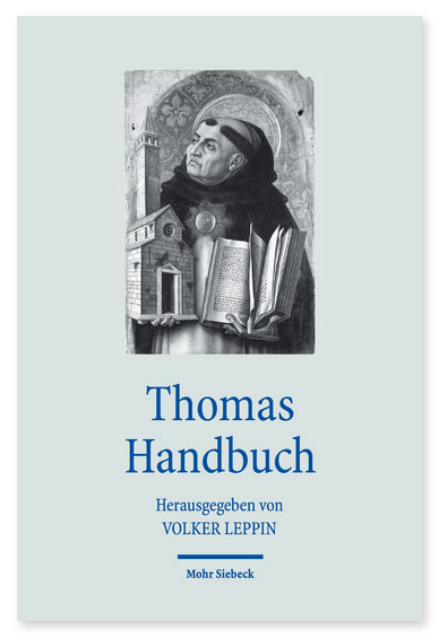

2016. XIV, 523 Seiten. HdTh

SBN 978-3-16-154297-8 DOI 10.1628/978-3-16-154297-8 eBook PDF 139,00€

ISBN 978-3-16-149230-3 fadengeheftete Broschur 49,00€ ISBN 978-3-16-150084-8 Leinen $139,00 €$
Das Thomas Handbuch bietet eine Darstellung von Leben, Werk und Wirkung von Thomas von Aquin, dem wohl bedeutendsten Vertreter der scholastischen Philosophie und Theologie in interdisziplinärer und internationaler Perspektive. Mit seinem Aufbau schließt es an die bewährten Theologen-Handbücher bei Mohr Siebeck an: In vier Sektionen bietet es Ausführungen zur Orientierung in der gegenwärtigen Forschungslage, zur Person des Thomas und ihren Verbindungen und Prägungen, zu seinem Werk und zu seiner Wirkung. Die Beiträge sind von Wissenschaftlern und Wissenschaftlerinnen aus den Bereichen Geschichte, Philosophie und - evangelische wie katholische - Theologie verfasst und repräsentieren die internationale Forschung. Das Thomas Handbuch bietet so eine Zusammenfassung der bisherigen Forschungen zu Thomas von Aquin und eine Grundlage für weitere Arbeiten. Es kann, erschlossen durch umfassende Register und Literaturangaben, als Nachschlagewerk genutzt werden, dessen einzelne Artikel je für sich verstehbar sind, aber auch als umfassende Gesamtdarstellung. Die interdisziplinären Beiträge eröffnen eine Gesamtschau, in welcher verschiedene Perspektiven ihren Ort finden und ihren je eigenen Beitrag zum Gesamten bieten. Angesichts der Bedeutung des Aquinaten kann es auch als Einführung in Grundlagen scholastischen Denkens genutzt werden.

Inhaltsübersicht

Teil A: Orientierung Henryk Anzulewicz: Handschriftliche Überlieferung, Werkausgaben - Henryk Anzulewicz: Hilfsmittel David Berger: Thomas-Forschung am Beginn des 21. Jahrhunderts

\section{Teil B: Person}

I. Stationen

Wolfgang Stürner: Kirche und Gesellschaft im 13. Jahrhundert - Volker Leppin: Domininkanerorden - Ingo Klitzsch: Scholastische Theologie: Lehrbücher und Inhalte - Volker Leppin: Köln, Paris und Neapel im 13. Jahrhundert - Gury Schneider-Ludorff: Das Leben des Thomas

II. Traditionen

Volker Henning Drecoll: Augustin - Reinhold Rieger: Dionysios Areopagita - Reinhold Rieger: Boethius - François-Xavier Putallaz: Lateinischer Aristotelismus - Philipp W. Rosemann: Petrus Lombardus - Hubert Philip Weber: Alexander von Hales und die Summa fratris Alexandri - Carmela Baffioni: Averroes und Avicenna - Jürgen Miethke: Papst und weltliche Mächte im 13. Jahrhundert - Elias H. Füllenbach: Dominikanische Spiritualität

\section{Beziehungen}

Mechthild Dreyer: Der Lehrer: Albertus Magnus - Marianne Schlosser: Der Kollege: Bonaventura - Ruedi Imbach: Die »Heiden« als Herausforderung - Volker Leppin: Bettelorden und Weltkleriker - Pieter de Leemans: Wilhelm von Moerbeke

\section{Teil C: Werk}

I. Schriften

Sabine Folger-Fonfara: De ente et essentia - David Berger: Quaestiones disputatae - Rolf Schönberger: Kommentare zu Boethius - Michael Basse: Sentenzenkommentar - Ruedi Imbach: Schriften gegen die pagane Philosophie und die konsequenten Aristoteliker - Reinhold Rieger: Kommentar zu Dionysios, De divinis nominibus - Thomas Prüg/: Bibelkommentare - Michael Basse: De rationibus fidei - Rolf Schönberger: Aristoteleskommentare - Sabine Folger-Fonfara: Kommentar zum Liber de causis - Jürgen Miethke: De regno ad regem Cypri - Ulrich Köpf: Summa Theologiae - Marianne Schlosser: Kleinere Schriften

\section{Themen}

Ulrich Köpf: Wissenschaftstheorie - Notger Slenczka: Gotteslehre - Christoph Schwöbel: Trinitätslehre - Reinhold Rieger: Gottesbeweise - David Berger: Schöpfungslehre - Notger Slenczka: Anthropologie - Notger Slenczka: Gnade und Rechtfertigung - Stephan Ernst: Theologische Ethik - Inos Biffi: Christologie - Marianne Schlosser: Sakramentenlehre - David Berger: Eschatologie

III. Strukturen

Volker Leppin: Theologie und Philosophie - Notger Slenczka: Theologie und Heil - Ulrich Köpf: Theologie als didaktische Aufgabe

\section{Teil D: Wirkung}

Elias H. Füllenbach: Seligsprechung - Peter Walter: Thomas im ersten Jahrhundert nach seinem Tod - Markus Wriedt: Reformatorische Auseinandersetzung - Peter Walter: Schulbildungen - Peter Walter: Neuthomismus

Volker Leppin Geboren 1966; Studium der Ev. Theologie; 1994 Promotion; 1997 Habilitation; 2000-10 Lehrstuhl für Kirchengeschichte in Jena; 2010-21 Lehrstuhlinhaber für Kirchengeschichte in Tübingen; seit 2021 Horace Tracy Pitkin Professor of Historical Theology an der Yale Divinity School.

https://orcid.org/0000-0001-8561-1086

Mohr Siebeck GmbH \& Co. KG

Postfach 2040

D-72010 Tübingen

info@mohrsiebeck.com

www.mohrsiebeck.com 
Jetzt bestellen:

https://mohrsiebeck.com/buch/thomas-handbuch-9783161542978?no_cache=1

order@mohrsiebeck.com

Telefon: +49 (0)7071-923-17

Telefax: $+49(0) 7071-51104$ 